\title{
Reservoir computing quality: connectivity and topology
}

\author{
Matthew Dale ${ }^{1,4}(1) \cdot$ Simon O'Keefe ${ }^{1,4} \cdot$ Angelika Sebald $^{2,4} \cdot$ Susan Stepney ${ }^{1,4} \cdot$ Martin A. Trefzer $^{3,4}$
}

Accepted: 12 November 2020/Published online: 15 December 2020

(C) The Author(s) 2020

\begin{abstract}
We explore the effect of connectivity and topology on the dynamical behaviour of Reservoir Computers. At present, considerable effort is taken to design and hand-craft physical reservoir computers. Both structure and physical complexity are often pivotal to task performance, however, assessing their overall importance is challenging. Using a recently developed framework, we evaluate and compare the dynamical freedom (referring to quality) of neural network structures, as an analogy for physical systems. The results quantify how structure affects the behavioural range of networks. It demonstrates how high quality reached by more complex structures is often also achievable in simpler structures with greater network size. Alternatively, quality is often improved in smaller networks by adding greater connection complexity. This work demonstrates the benefits of using dynamical behaviour to assess the quality of computing substrates, rather than evaluation through benchmark tasks that often provide a narrow and biased insight into the computing quality of physical systems.
\end{abstract}

Keywords Reservoir computing · Unconventional computing · Topology $\cdot$ Connectivity $\cdot$ Dynamical behaviour

\section{Introduction}

Reservoir Computing (RC) (Schrauwen et al. 2007; Verstraeten et al. 2007) is a computational model used to train and exploit an increasingly rich variety of dynamical systems, ranging from virtual neural networks to novel physical systems, such as quantum, electrical, chemical, optical and mechanical [see reviews by Lukoševičius and Jaeger (2009), Tanaka et al. (2019)]. Every reservoir system is designed to harness the underlying dynamics of the substrate it is implemented with, whether that be a physical device or a simulated dynamical system. However, finding a suitable substrate, or designing one, with sufficient dynamics to compute specific tasks is challenging.

Matthew Dale

matt.dale@york.ac.uk

1 Department of Computer Science, University of York, York, UK

2 Department of Chemistry, University of York, York, UK

3 Department of Electronic Engineering, University of York, York, UK

4 York Cross-disciplinary Centre for Systems Analysis, University of York, York, UK
Methods to assess the range of dynamics a substrate can exhibit are undeveloped. Therefore, matching substrate to task is typically done through trial and error. This often involves a long and laborious exercise to determine how best to configure and design a substrate to improve performance.

Dale et al. (2019b) present a framework to assess and compare computing systems based on abstract behaviours of dynamical properties. Using a Quality-Diversity algorithm (Pugh et al. 2016), the full dynamical range of the substrate is mapped and explored. The characterisation of the substrate is then used to determine the effects of configuration, perturbation and design alteration on substrate "quality". Dale et al. (2019a) use the framework to investigate the role of connection types and topology on the quality of simulated recurrent networks. They find that an increase in network size can compensate for a reduction in network complexity, and vice versa.

Here we perform an extension of that work, with more network sizes, longer searches, more runs, and different restrictions to network creation and alteration: no topological connections in the underlying network can be broken, and a constant number of connections is maintained during the exploration process. We additionally investigate 
what effect adding extra random connections has on the quality of each topology.

Simple topologies are typically easier to implement in hardware. If adding only a few additional connections leads to higher quality, there is the potential for new computing capabilities with minimal cost in hardware. Here we explore whether adding only a few additional connections to regular topologies can lead to more dynamical behaviours, behaviours typically accessible only to networks with higher degrees of freedom, for example, with many more connections.

In the reservoir computing literature, small-world topologies-with both regular structure and random connectivity (Deng and Zhang 2007; Kawai et al. 2019) have been assessed on multiple benchmark tasks. It was found that small-world reservoir networks often improve task performances and provide greater robustness in parameter selection. However, it is unknown how this topology affects quality, e.g., does it provide the same, less or more dynamical freedom? What happens to reservoir quality as we increase the connectivity of regular networks towards fully-connected networks? Here, we address both of these questions and discover that small-world-like connectivity does not lead to any greater quality and that the quality of regular structures tends to converge to fullyconnected networks with an additional $2.5-5 \%$ connections.

\section{Reservoir computing}

Exploiting the intrinsic properties of physical systems has the potential to offer improvements in performance, efficiency and/or computational power compared with conventional computing systems (Crutchfield 1994; Lloyd 2000). However, to do so requires a model of computation that naturally fits the substrate rather than imposing a model that fights its implementation (Stepney 2008).

The simplicity and black-box exploitation of the reservoir computing model means that it suits many types of physical systems. However, its simplicity has drawbacks, for example, it struggles to solve complex tasks requiring high-order abstractions and multiple timescales (Gallicchio et al. 2017; Lukoševičius and Jaeger 2009).

Input-driven reservoir computers are typically represented and divided into three layers: the input, the reservoir, and the readout. The reservoir is the dynamical system, and perturbed and observed as a black-box. The input and readout depend on the chosen method to encode and decode information to and from the reservoir. Depending on the implementation, this could be serial or parallel input-outputs, and discrete or continuous values encoded as electrical, optical, chemical, mechanical or other signals.
Each reservoir is configured, controlled and tuned (programmed) to perform a desired function. This often requires the careful tuning of parameters in order to produce working and optimal reservoirs. Most reservoirs are hand-crafted to a task, typically requiring expert domain knowledge to design an optimal system. However, the separation between layers allows the reservoir to be optimised and configured independently of the input and readout layer. Many techniques have been used to optimise virtual reservoirs (Bala et al. 2018) and physical reservoirs (Dale et al. 2016a, b, 2017).

To interpret a substrate as a reservoir, we define that the observed reservoir states $x(n)$ form a combination of the substrate's implicit function and its $n$th discrete observation:

$x(n)=\Omega\left(\mathcal{E}\left(W_{\text {in }} u(t), u_{c}(t)\right)\right)$

where $\Omega(\cdot)$ is the observation of the substrate's macroscopic behaviour and $\mathcal{E}(\cdot)$ the continuous microscopic substrate function, when driven by the input $u(t)$ at timestep $t . W_{\text {in }}$ is the input connectivity to the reservoir system; this is typically represented as a random weight matrix (see Lukoševičius (2012) for a guide on creating reservoir weights). The variable $u_{c}(t)$ represents the substrate's configuration (program), whether that be through external control, an input-output mapping, or other method of configuration. Typically, $u_{c}(t)$ is not a function of time, but only of a given problem, for example, the hyperparameters in simulated networks, or bias voltages in electronic systems. However, modulating or switching between different configurations over time could add additional dynamical complexity.

The final output signal $y(n)$ is determined by the readout function $g$, on the observation $x(n)$ :

$y(n)=g(x(n))$

Note that the intrinsic function $\mathcal{E}(\cdot)$ of the substrate is described as being fixed, clamped, or set by $u_{c}$; only $g$ is adapted. However, depending on the system, $\mathcal{E}(\cdot)$ may change when interacted with or observed, and therefore be non-deterministic. Additionally, $\mathcal{E}(\cdot)$ may also have a stochastic nature and be influenced by its environment; appropriate sampling and averaging are required in these cases.

The observation function $\Omega(\cdot)$ is also considered fixed, but could change depending on the observation method and equipment.

This formalisation separates the system into contributing parts, including the observation and configuration method. This is key to understand for physical reservoir computing-every element represents the reservoir system, not just the substrate-leading to more ways to improve, optimise and exploit. 


\section{CHARC framework}

CHARC (CHAracterisation of Reservoir Computers) (Dale et al. 2019b) provides a framework with which to explore and compare the dynamical and computational expressiveness of computing substrates. The expressiveness of a substrate is determined by how many distinct dynamical behaviours it can exhibit. The total number of behaviours is then used to approximate the quality of the substrate to perform reservoir computation.

Through this process (see Fig. 1 for workflow), the applied computational model, e.g., encoding, decoding and abstract representation of the system, is also assessed, suggesting whether the chosen model is a suitable fit to the physical implementation.

To characterise the computing quality of substrates, the framework searches over a set of metrics measuring favourable dynamical properties. At present, these metrics are targeted at properties critical to reservoir computing, however, the framework is not limited to these. If a substrate is able to exhibit many dynamical behaviours, it is said to have a greater quality and therefore a increased capacity to solve different classes of computational problems.

Dale et al. (2019b) use CHARC to explain why evolved configurations of disordered carbon nanotube composites perform well at certain tasks and not others: due to their limited dynamical behaviour. They also show that delayline reservoirs (Appeltant et al. 2011) often match the dynamics of large simulated recurrent neural networks, partly explaining the competitiveness of such systems.

The basic CHARC framework is designed with various levels to define and measure quality. The output of lower levels are used by higher levels to model relationships between parameters, dynamics and task performance.

At the base level, the computational model is chosen. The reservoir computing model is applied here. In the context of a model, an abstract space of behaviours is defined. This space represents the dynamical behaviour of the substrate when configured. This space is distinct from the substrate configuration space, where small changes in configuration parameters can result in large changes in behaviour, and vice versa.

To define this $n$-dimensional behaviour space, $n$ independent property measures are used. Here, we use the same three-dimensional behaviour space used in Dale et al. (2019b), using three metrics measuring basic properties required for reservoir computing: Kernel Rank (KR; the separation property, Legenstein and Maass 2007), Generalisation Rank (GR; the generalisation property, Legenstein and Maass 2007), and Memory Capacity (MC; the echo state property, Jaeger 2001). An example 3-d behaviour space is shown in Step 1 of the CHARC workflow, Fig. 1 .

For an outline of how to implement these measures consult Dale et al. (2019b). As mentioned there, these three measures by themselves do not fully capture all dynamical properties of a reservoir. To improve accuracy and the quality measure, additional independent property measures may be required. However, these three measures are generally sufficient to demonstrate the framework and evaluate the various substrates proposed here.

The Exploration \& Mapping level of the framework defines the search method used to construct the mapping between abstract reservoir and substrate configuration. This is shown as Step 2 in Fig. 1. The behaviour space is explored using an adapted implementation of novelty search (Lehman and Stanley 2008), an open-ended genetic algorithm that explores a search space for novel solutions until a user-defined termination criterion is met.

Novelty search discovers a wider range of behaviours than does random search (Dale et al. 2019b) for the same number of evaluations. CHARC maps the entire behaviour space, not just boundaries, and hence novelty search is a more appropriate search technique than explicit optimisation of some objective function. This mapping process, by covering the full space, can determine the practical use of

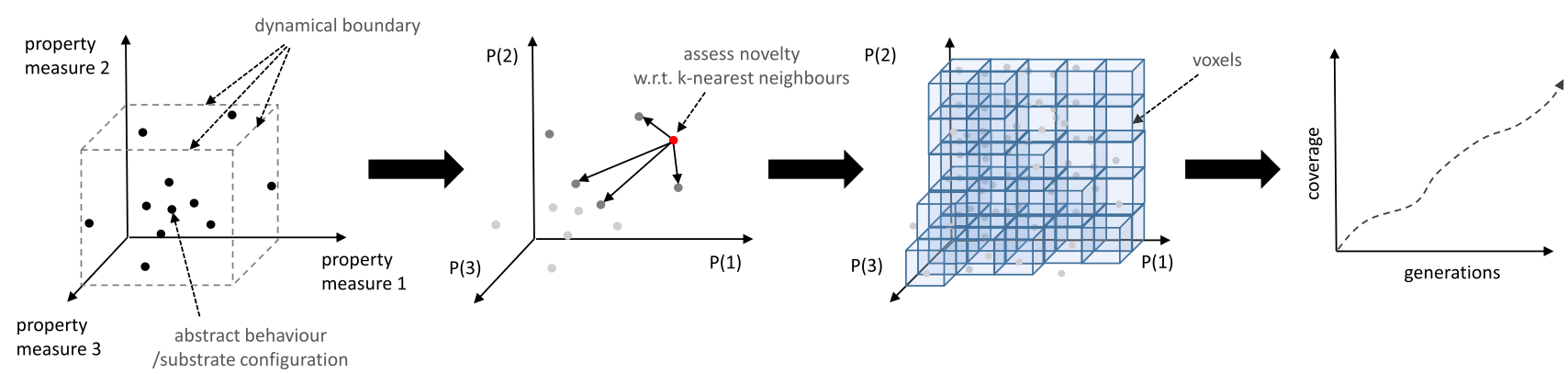

Fig. 1 CHARC framework basic workflow 
the substrate, or whether the computational model and configuration method is appropriate.

The use of heuristic search, rather than exhaustive search, may result in some biases in the results. For example, the discovered boundaries of the behaviour space are noisy as they are the most difficult behaviours to find, therefore they are less frequently discovered. The discovered boundaries also tend to be noisier for systems with higher quality (see Fig. 4) as the boundaries of lower quality systems are easier to find in fewer search iterations. The quality measure used compensates for some noise: if a behaviour is found, all nearby behaviours are taken to be possible; CHARC provides a qualitative approximation of reservoir quality. Also, different reservoir architectures will have different biases: although some bias is removed because of the focus on the resulting behaviour space instead of configuration parameter space, nevertheless a larger number of configuration parameters will require greater exploration, and different architectures have different parameter combinations. The fairest way to compare is the standard approach taken in using evolutionary algorithms: put the same restriction on the length of each search. Some architectures may in principal be underexplored, but the results indicate that in fact there is reasonably consistent coverage of behaviour space.

The Evaluation level defines the mechanisms to evaluate quality. This constitutes the final level for measuring quality. To assess quality, the final behaviour space is divided into voxels/cells as demonstrated in Step 3 of Fig. 1. The total number of voxels that are occupied by behaviours builds an approximation of the dynamical freedom: how many distinct reservoirs the substrate can instantiate. This acts as the measure of quality to compare across systems.

In order to compare fairly across systems the same number of evaluations and dimensions should be kept, as well as the same voxel size when measuring quality.

\section{Network topology experiments}

Simple, regular and deterministic connection topologies can perform as well as randomly-generated reservoir networks on a number of benchmark tasks (Appeltant et al. 2011; Ortín et al. 2015; Rodan and Tiňo 2010, 2011). However, the exact role and benefits of complex structures in reservoir computing is an open question and the full computing and dynamical limitations of simple topologies still remains unclear.

\subsection{Reservoir model}

The dynamics of each reservoir network used in this work is given by the state update equation:

$$
x(n)=(1-a) x(n-1)+a f\left(b W_{i n} u(n)+c W x(n-1)\right)
$$

where $x$ is the internal state at time-step $n, f$ is the neuron activation function (a tanh function), $u$ is the input signal, $W_{i n}$ and $W$ are weight matrices giving the connection weights to inputs and internal neurons respectively. The parameters $a, b$ and $c$ control the global scaling of: leak rate, input weights and internal weights. The leak rate controls the time-scale mismatch between the input and reservoir dynamics; when $a=1$, the previous states do not leak into the current states. Input scaling $(b)$ affects the non-linear response of the reservoir and relative effect of the current input. Internal scaling $(c)$ controls the reservoirs stability as well as the influence and persistence of the input; e.g. low values dampen internal activity and increase response to input, and high values lead to chaotic behaviour.

The final trained output $y(n)$ is given when the reservoir states $x(n)$ are combined with the trained readout weight matrix $W_{\text {out }}$ :

$$
y(n)=W_{\text {out }} x(n)
$$

\subsection{Topologies}

In the experiments here, we use CHARC to investigate the effect of network topology on quality by evaluating four simulated reservoir networks: ring, lattice, torus and fullyconnected networks.

The ring topology (Fig. 2a) has the least complexity. Each node has a single self-connection and a weighted connection to each of its neighbours to its left and right, resulting in every node having three adaptable weights in the internal weight matrix $W$.

The ring is the simplest network to implement in physical hardware as the number of connections required is small. Ring structures with various connection types have been applied to many reservoir computing systems, including Simple Cycle Reservoirs (SCR) (Rodan and Tiňo 2011), DNA reservoirs (deoxyribozyme oscillators) (Goudarzi et al. 2013), and delay-line reservoirs using a single non-linear node with a delay line (Appeltant et al. 2011; Paquot et al. 2012).

The lattice topology (Fig. 2b) has greater connection complexity. With this topology, we define a square grid of neurons each connected to its nearest neighbours [using its Moore neighbourhood, as commonly used in cellular 


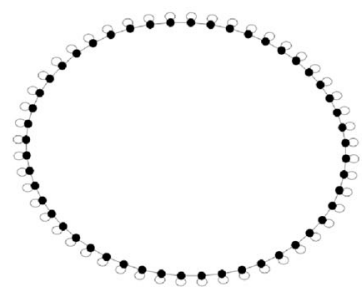

(a) ring

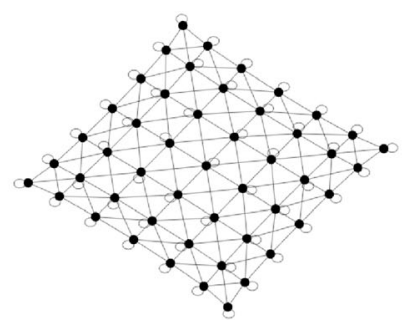

(b) lattice

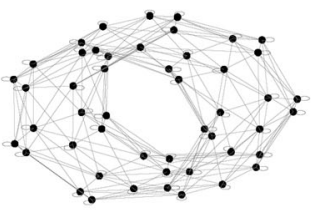

(c) torus

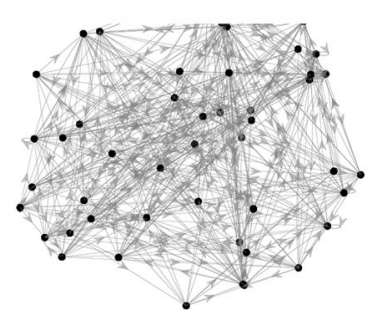

(d) Fully-connected

Fig. 2 Network topologies under investigation

automata (Adamatzky 2010)]. Each non-perimeter node has eight connections to neighbours and one self-connection, resulting in each node having a maximum of nine adaptable weights in $W$.

Lattice networks/models are common in computational physics, condensed matter physics and beyond, modelling physical interactions, phase transitions and structure (Lavis 2015). Examples include: discrete lattices like the Ising model with variables representing magnetic dipole moments of atomic spins (Brush 1967), and the Gray-Scott reaction-diffusion model to simulate chemical systems (Pearson 1993). Physical substrates often have a regular grid of connections; lattice networks are therefore a realistic representation of many physical systems that would be considered for reservoir computing.

The torus topology (Fig. 2c) is a special case of the lattice where perimeter nodes are connected to give periodic boundary conditions. Each node has nine adaptable weights in $W$.

Although not a natural topology for physical systems, turning physical lattices into tori is often relatively simple. An advantage of the toroidal structure is that it reduces the signal path distance between perimeter nodes and removes edge effects, leading to potentially different dynamics at a minimal cost to connection complexity.

The fully-connected network (Fig. 2d) has no topological constraints and has the maximum number of adaptable weights: the weight matrix $W$ is fully populated. This network type is used to benchmark the other topologies. Due to its large degree-of-freedom in parameter space, this network likely features the highest quality with respect to its size. However, fully-connected and random networks are challenging to implement in physical hardware due their connection complexity.

Extra complexity can be achieved by increasing the number of nodes and weights. The number of non-zero weights in $W$, as a function of the number of nodes $N$ is shown in Table 1.
Table 1 The number of non-zero weights in $W$, as a function of the number of nodes $N$, for the four topologies

\begin{tabular}{ll}
\hline Topology & No. of weights \\
\hline Ring & $3 N$ \\
Lattice & $9 N-12 \sqrt{N}+4$ \\
Torus & $9 N$ \\
Fully-connected & $N^{2}$ \\
\hline
\end{tabular}

\subsection{Experimental parameters}

To measure the effect topology has on dynamical behaviour and quality, we test each topology across several network sizes. The network sizes range from $4^{2}=16$ to $20^{2}=400$ nodes. By comparing the different sizes, we can determine what relationships exist independent of size, and how relationships scale with network size.

Each network has local (e.g., weights) and global (e.g., weight scaling) parameters under manipulation during the search process. Individual weights in the input layer matrix $W_{\text {in }}$ and the reservoir matrix $W$ are open to mutation and can be mutated between $[-1,1]$. Global scaling parameters $(a, b$ and $c$ ) are also evolved. For internal weight $W$ scaling this is a value between $[0,2]$, and for $W_{i n}$ scaling between $[-1,1]$. The leak-rate parameter, controlling the "leakiness" of past states into current states, is restricted between $[0,1]$. Input and internal connectivity of the reservoir remains constant, depending on the topology. The weight distribution of $W_{\text {in }}$ and $W$ is evolved by a Gaussian mutation operator.

The output weight matrix $W_{\text {out }}$ for each network is used only for the memory capacity measure, as both KR and GR are calculated using only the reservoir states. When the readout layer $W_{\text {out }}$ is in use, training is carried out using ridge regression to minimise the difference between the network output $y$ and the target signal $y_{t}$.

The CHARC parameters used in the experiments here are given in Table 2 (see Dale et al. (2019b) for more 
Table 2 Search algorithm parameters for all experiments

\begin{tabular}{ll}
\hline Parameter & Value \\
\hline Runs & 20 \\
Generations & 10,000 \\
Population size & 100 \\
Deme size & $20 \%$ \\
Mutation rate & $10 \%$ \\
Recombination rate & $50 \%$ \\
$\rho_{\text {min }}$ & 3 \\
$K_{\text {neighbours }}$ & 10 \\
$\rho_{\text {min }}$ update & 100 generations \\
Voxel size & 10 \\
\hline
\end{tabular}

information). Novelty search is applied using an adapted version of the steady-state microbial genetic algorithm (Harvey 2009). Using this algorithm, each generation produces one new individual that is measured for novelty.

The parameters deme size and recombination rate refer to division of species in the population and percentage of genes to inherit. The parameters $\rho_{\min }, K_{\text {neighbours }}$ and $\rho_{\min }$ update control the novelty measure.

To produce a good estimate of quality, typically a large number of novelty search generations are needed. In practice, the number used depends on computing resources and time available to evaluate each reservoir. As shown in Dale et al. (2019b), a rough estimate is typically achieved with less than a few thousand search generations. Here, we apply 10,000 generations to create a detailed picture of each topology and its role.

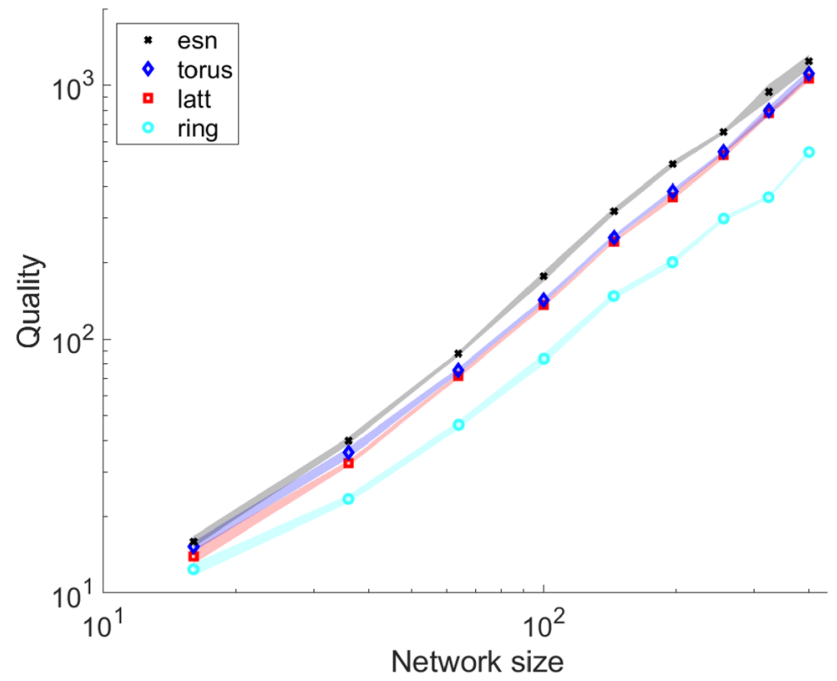

Fig. 3 Mean quality (dots) and standard deviation (shaded area) of all network topologies, for network sizes $N=4^{2}=16,6^{2}=36$, $8^{2}=64, \ldots, 20^{2}=400$ nodes $(\log -\log$ plot $)$
In all experiments a voxel size of 10 is used for the quality measure, leading to a voxel volume of $10^{3}$ for the 3-dimensional behaviour space.

Each novelty search run begins with a random initial population. 20 separate runs are conducted for each network size and topology, to gather statistics of quality.

\subsection{Results}

The results for topology and network size are given in Fig. 3. As network size increases, general network capacity increases, as expected. The variation (shaded area) between runs is similar across all the regular topologies. The fullyconnected network tends to have greater variation across runs than the others, indicating a stronger dependency on the initial random population.

At a given size (number of nodes) the fully-connected networks occupy a greater volume of the behaviour space, possessing a higher quality, than the others, across all sizes. Also, lattices and tori, with an intermediate number of connections between rings and fully connected networks, have intermediate quality. This suggests that, for a fixed number of nodes, access to more adjustable parameters (table 1) typically leads to more dynamical behaviours being available (see $\$ 4.4 .2$ for more on this).

The lattice and torus have similar qualities across all sizes; however, the difference between them is statistically significant. Using the non-parametric Wilcoxon rank sum test, the difference at all sizes (except at $N=324$ nodes, with $p=0.053$ ) is significant at the $95 \%$ confidence level $(p<0.05)$.

The quality of these two topologies is closer to the fullyconnected network than the ring topology. This suggests there are only a few behaviours the fully-connected can exhibit that these two topologies cannot.

As size increases, the quality of the ring topology increases more slowly than the others. To exhibit a similar quality to the others would require an approximate doubling of ring size.

\subsubsection{Behaviour space}

To understand what these differences in quality represent, we visualise the 3 -d behaviour space as three projections. The behaviour space of each topology is shown separately in Fig. 4a-d, with each network size superimposed onto the same space. The largest network is placed at the back with each network size layered in decreasing order towards the smallest network in the front. Each network size is also separated by colour.

In Fig. 4, some common patterns exist for each topology. Smaller networks are more efficient in terms of 

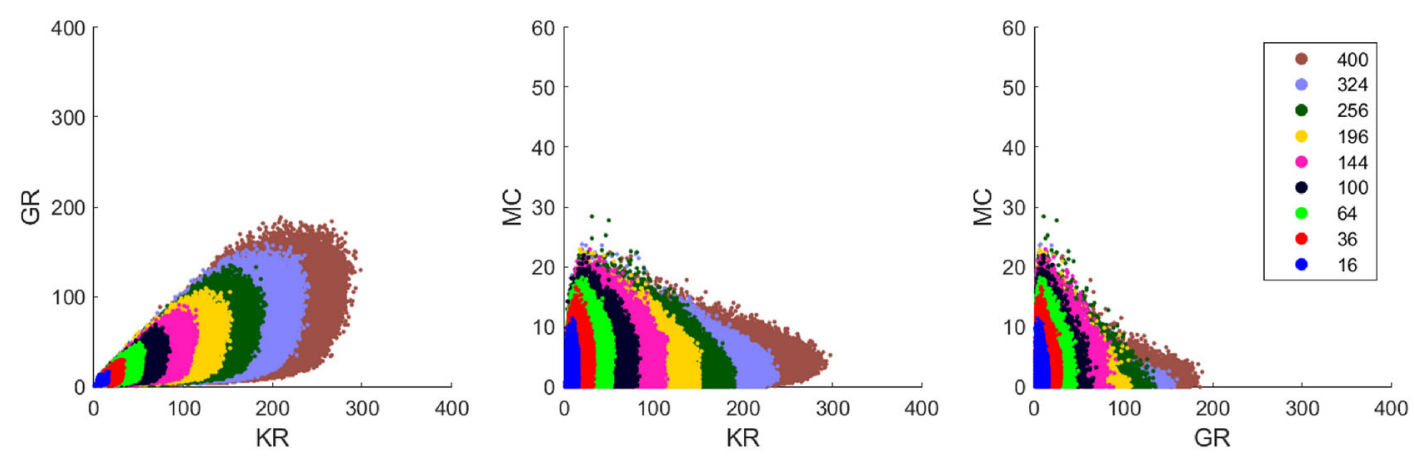

(a) Ring
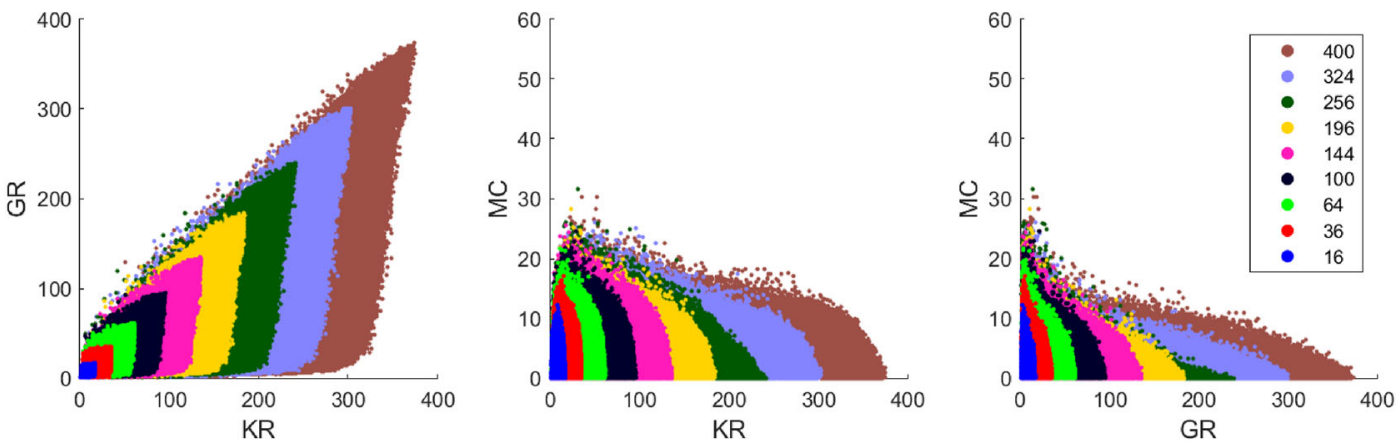

(b) Lattice
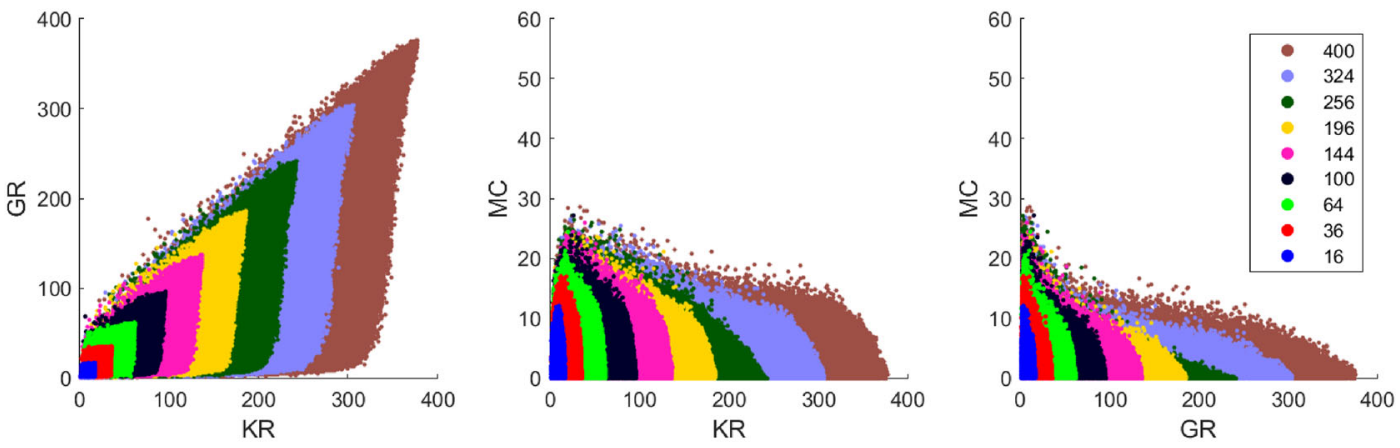

(c) Torus
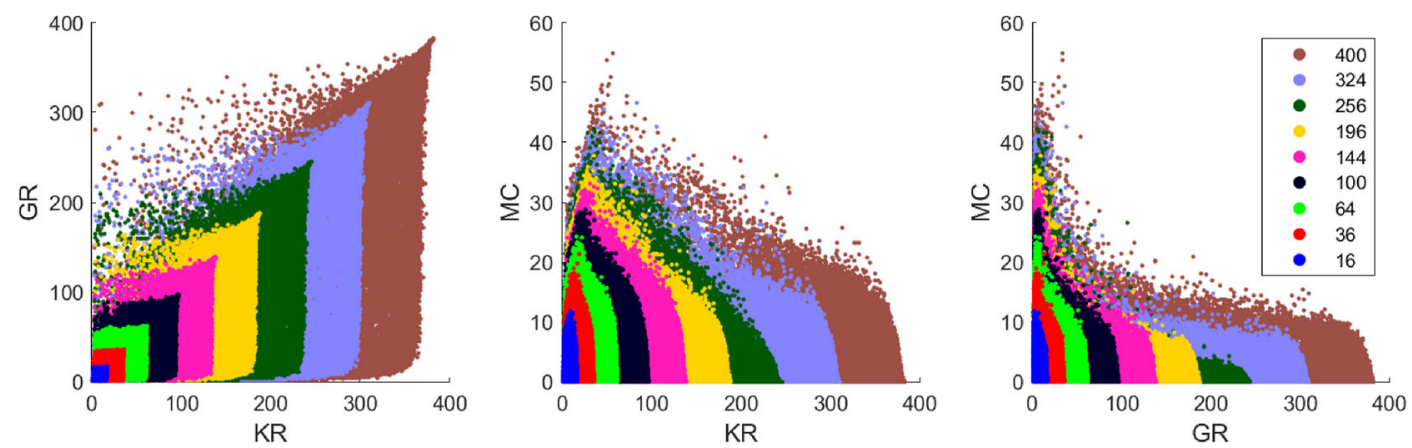

(d) Fully-connected

Fig. 4 Behaviour spaces of all topologies and network sizes after 10,000 generations and 20 runs. A total of 200,000 network behaviours are shown for each network size 
memory capacity-i.e., possess a larger memory capacity with respect to network size. As size increases, there is a considerable drop in efficiency with little gain in overall capacity.

Sparse areas of the behaviour space highlight challenging properties to all reservoir structures. For example, the regions where trade-offs between non-linearity (high $\mathrm{KR}$ ), noise (high GR) and memory (high MC) are most challenging.

The ring topology (Fig. 4a) tends to exhibit more linear and ordered dynamics characterised by smaller KR and GR values. As size increases, the space occupied proportional to network size tends to decrease. This implies that larger networks struggle more than smaller networks to maintain non-linearity and input separability.

In terms of non-linear and chaotic dynamics, the lattice and torus topology (Fig. 4b, c) scale relatively well with size. Yet, only small gains are made in memory capacity as size increases; some outliers suggest larger MC values are possible (longer novelty search runs could discover if this is the case). This limitation to memory capacity explains why both topologies have less quality than the fully-connected network. The difference between the torus and the lattice is due to more consistent high memory capacities across runs, as seen by the slightly more densely filled areas in Fig. 4c.

The fully-connected networks (Fig. 4d) do not feature the same memory limitations as the regular topologies. This network-type frequently exhibits the more challenging behaviours, occupying regions with greater trade-offs between non-linearity and memory. Interestingly, these networks can occupy areas where noise increases (GR) but input separation (KR) does not: the upper-left region of the KR-GR space. This trade-off is more prominent in the fully-connected network.

\subsubsection{Number of connections}

Our results above suggest that access to more adjustable parameters (non-zero elements in $W$ ) typically leads to more dynamical behaviours. Here, we investigate this further.

The number of adjustable weights of a given network is determined by the network size and topology (table 1), and is constant during the evolutionary search. We show quality versus the number of adjustable weights in Fig. 5. For each topology, there appears to be power-law scaling with respect to quality and the number of adjustable weights.

As the number of adjustable weights affects network complexity and the size of the configuration space, we expect an increase in degrees-of-freedom and possibly behavioural freedom with increase in number of weights.
However, there are subtleties. The order of the lines in Figs. 3 and 5 are reversed. So, at a given number of $a d$ justable weights, the fully-connected networks occupy a smaller volume of the behaviour space, possessing a lower quality, than the others, across all sizes; they also possess many fewer nodes. Also, lattices and tori, with an intermediate number of connections between rings and fully connected networks, have intermediate quality. This suggests that, for a fixed number of adjustable weight parameters, access to more nodes (table 1) typically leads to more dynamical behaviours being available.

\subsubsection{Balancing nodes and connections}

The different effects of more weights or more nodes can be explained by the fact that some behaviour metrics (KR and GR) are bounded by the number of nodes, and that bound is relatively easy to achieve (Fig. 4). So more adjustable weights allow fuller exploration of the available behaviour space, and hence higher achievable quality, whereas more nodes allow a larger dynamical behaviour range, and hence the potential for higher quality if that range can be exploited. Fully connected networks have a maximum number of weights for a given number of nodes, and hence are the most fully explorable for that number of nodes; rings have a maximum number of nodes for a given number of weights, and hence have the largest potential behaviour space for that number of weights.

Based solely on an inspection of Figs. 3 and 5, quality can be maintained by a switch of topology, adding more nodes, and reducing the number of connections. This may

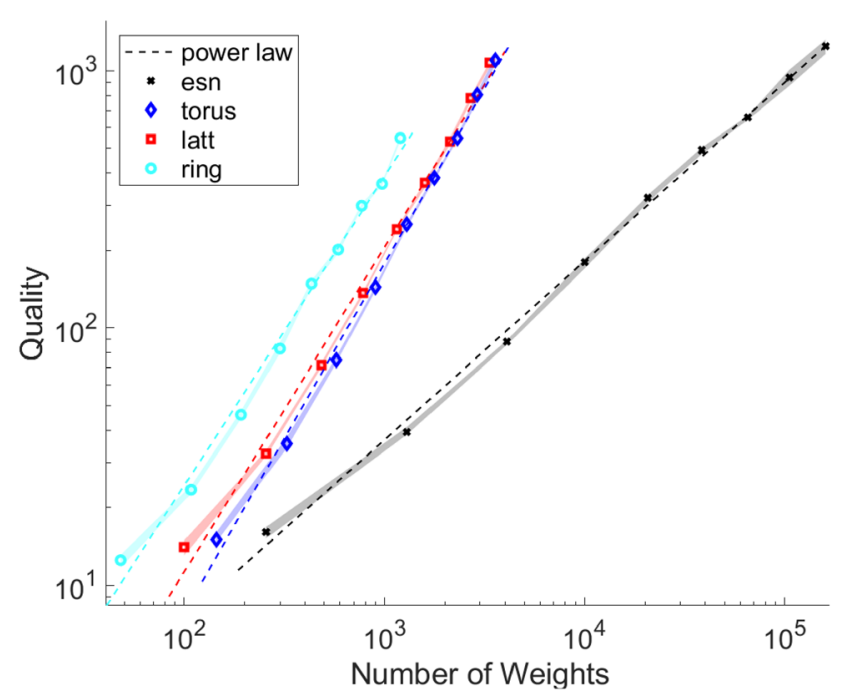

Fig. 5 Relationship between number of adjustable weights and quality for all networks (log-log plot). Each topology is represented by its respective symbol and colour. The points on each topology line are the network sizes evaluated: (bottom to top) 16, 36, 64, 100, 144, 196, 256, 324 and 400. (Color figure online) 
Fig. 6 Example 64-node networks with a ring topology and percentage of additional connections. Colour represents strength and sign of weighted connections. (Color figure online)

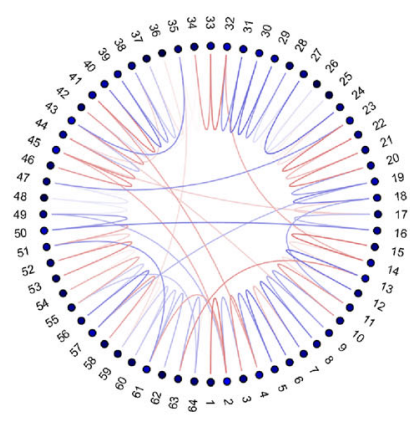

(a) $1 \%$

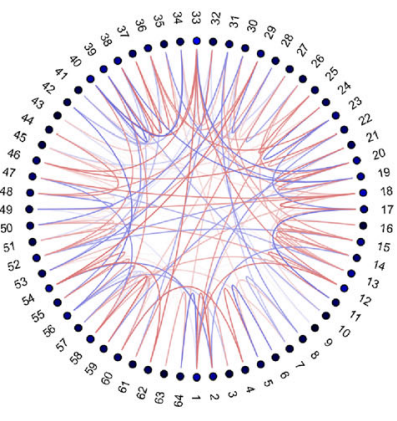

(b) $5 \%$

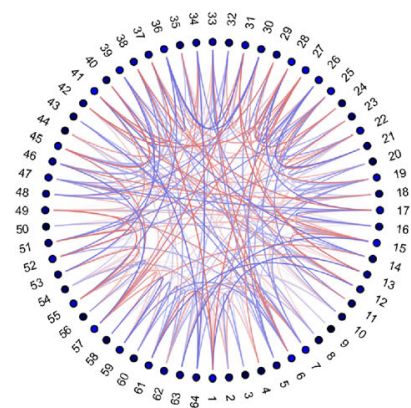

(c) $10 \%$ have advantages for manufacturing reservoir substrates. However, the quality value is a somewhat crude measure, and may not result in the same collection of behaviours being produced, for example, different systems with the same overall quality might not be able to achieve the same memory capacities (see Fig. 4). The quality measure alone is not sufficient to guarantee performance on specific tasks: it gives the overall potential of a substrate, but different tasks require different values of behavioural metrics (Dale et al. 2019b) that may lie inside or outside the overall quality envelope.

\section{Network connectivity}

Rather than being regular or completely random, many interesting biological, technological and physical networks are said to feature small-world topologies or scale-free properties, exploiting both regular and random structure (Watts and Strogatz 1998). Networks are considered small-world when they have a small average path-length between any two nodes and a high clustering coefficient. Scale-free networks are characterised by an asymptotic degree distribution among nodes with large 'hub' nodes typically followed by many smaller-hub nodes.

Both scale-free and small-world topologies have been applied to Echo State Networks and have shown to improve performance across certain benchmark tasks (Deng and Zhang 2007; Kawai et al. 2019). However, the full range of behaviours exhibited by small-world networks may be limited. Kawai et al. (2019) find that small-world reservoirs could not replicate the high linear memory capacities produced by random and regular networks.

In the experiment in this section, we add a fixed quantity of evolvable connections to the previous regular structures, to assess their affect on quality. Adding extra connections to each topology reduces the signal path length between distant nodes and alters the degree distribution of nodes in each topology. We increase the total number of connections for each topology by setting a randomly-selected percentage of the non-topological weights-all otherwise zero-valued entries in $W$. These new networks, each with the original topology plus extra random connections, are then assessed for quality. An example of the ring topology with extra connections is shown in Fig. 6. $100 \%$ additional connections to any topology results in a fully-connected network.

Unlike the Watts and Strogatz (1998) small-world model, which uses a set rewiring probability and set degree for each node, or the Barabási and Albert (1999) scale-free model, which applies preferential attachment, we allow evolution to rewire and converge to any degree distribution. The total number of connections remain constant for each network, as in previous experiments; we fix the percentage of extra connections, but allow evolution to chose which particular connections are used. Whether a new connection is local or distant and what degree each node has is left to evolution to decide. As before, mutation can still change individual weight strengths and global network parameters.

Figure 7 shows quality versus connectivity of all three topologies at five network sizes. Figure 7 (left) shows percentage of additional connections versus quality and Fig. 7 (right) shows number of weights against quality. The percentage of connections in both plots begins at $0.05 \%$ and increases to $100 \%$ (a fully-connected network). The mean quality is given over 10 runs with standard deviation represented by the shaded area. Colours and symbols represent each topology; lighter and darker shades are used to separate network sizes.

The lattice and torus increase only marginally in quality with more connections. The ring exhibits a significant improvement in quality until it converges with the other topologies at around 2.5-5\%. Depending on network size, this convergence occurs roughly when, or slightly before, the number of extra connections equals the number topology connections. This symbolises the transition point when the dominant structure shifts from given topology to unstructured network. 


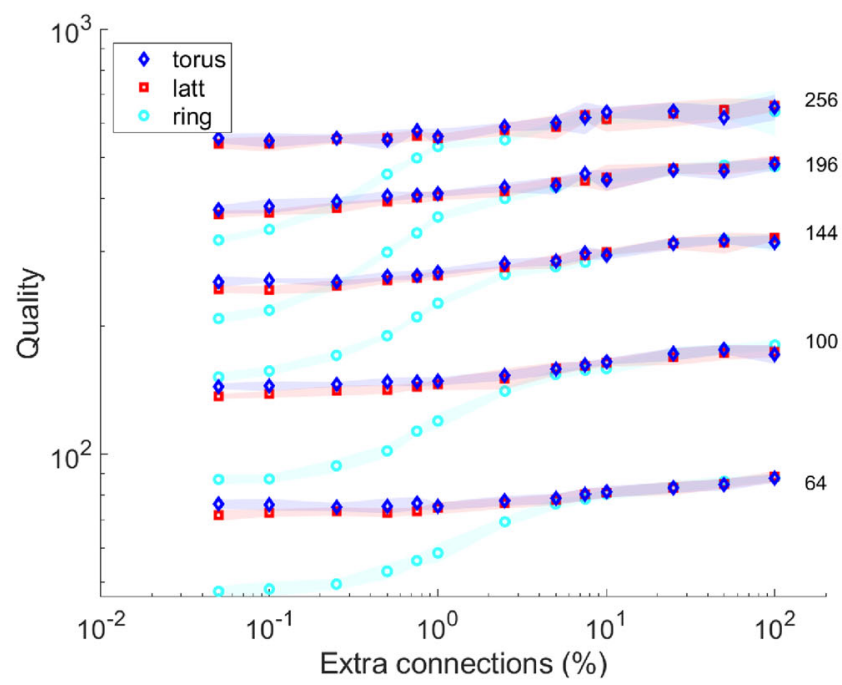

Fig. 7 Topology, network size and percentage of extra connections ( $\log -\log$ plot). The quality of each topology with additional connections, across the five sizes: 64, 100, 144, 196 and 256 nodes.

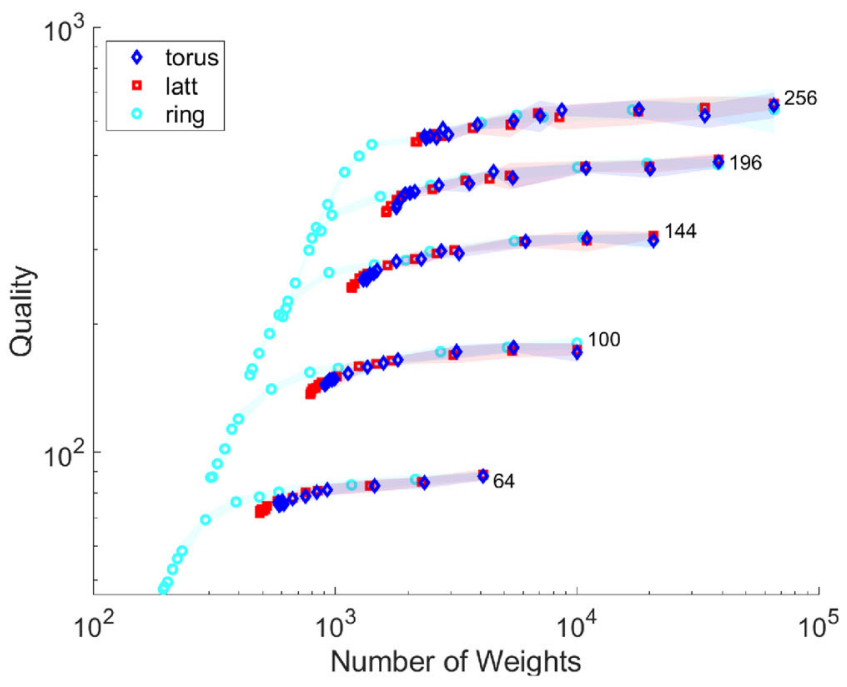

(left) percentage of extra connections: $0.05-100 \%$; (right) total weights of topology with extra connections
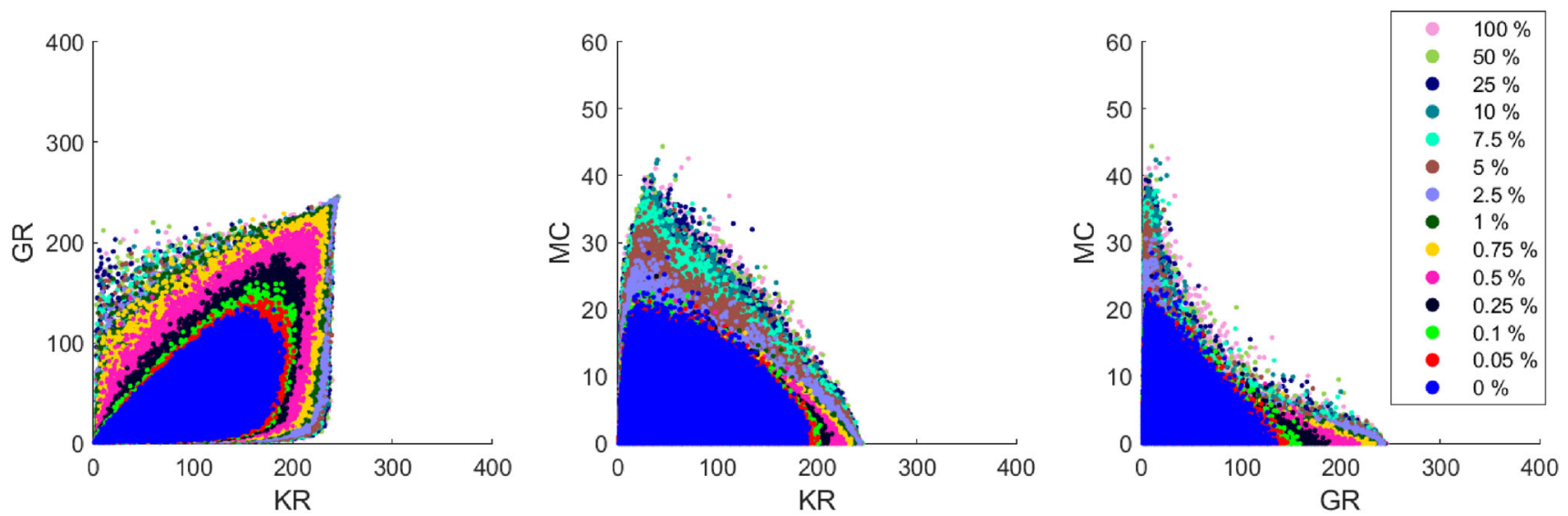

Fig. 8 Behaviour space of 256-node ring topology with additional percentage of connections

Sparse networks-particularly between 1 and $10 \%$ connectivity-exhibit approximately the same quality as fully-connected networks. An advantage of sparse connectivity is a linear scaling in computational complexity to compute as size increases.

To further understand the role of connectivity, we examine the behaviour space of the ring topology at 256 nodes, Fig. 8. Here, the discovered behaviours of all rings with different connectivity are layered, starting with the original ring topology $(0 \%)$ at the front, then increasing in connectivity towards the back $(100 \%)$.

In the KR-GR space (left), the most notable new behaviours appear between 0.1 and $2.5 \%$. After this, there are few new behaviours as the bounds of KR and GR are approached. In the KR-MC space (middle), greater coverage is seen between 1 and $25 \%$; memory capacity grows by roughly $50 \%$ between 1 and $5 \%$ of extra connections. Beyond $25 \%$, there is relatively small, if any, improvement in MC. In the GR-MC space (right), the inverse relationship between both properties is seen: a high MC requiring a low GR. For GR, an increase in connectivity appears to affect coverage incrementally, however, MC only increases at the higher connection percentages.

\section{Conclusion}

Assessing and comparing how structure affects dynamical behaviour is challenging. For unconventional substrates, determining what predefined structure exists and what part is useful is even more challenging. Even if structure can be 
decided at creation, what is a suitable or ideal structure is often limited by physical constraints.

In this work, we have assessed what effect different structural choices have on quality and how reservoir networks might be improved with minimal cost to connectivity. We have applied the task-independent CHARC framework and used it to directly assess topology and connectivity and their role on the computing quality of recurrent neural networks. The results show that networks with simple topologies and sparse connectivity exhibit similar quality and behaviours as more complex structures. However, simple topologies, such as the ring, tend to have limited behaviour. To overcome this, we propose adding extra connections to each topology. The results indicate that quality can increase with extra connections to each topology, with the ring topology being the most affected. At around 2.5-5\% extra connectivity, each topology tends to converge towards the same quality across all network sizes. After this, only minor improvements are found. This point of convergence typically coincides with the number of extra connections matching, or outnumbering, the connections required to form each topology.

Our interpretation of these results is that small-worldlike connectivity does not lead to any greater dynamical behaviour or freedom than fully-connected networks. However, quality is clearly improved when the topological constraints are relaxed.

In future work, we will apply these design principles and methods to create better physical computing substrates.

Acknowledgements This work is part of the SpInspired project, funded by EPSRC Grant EP/R032823/1. All experiments were carried out on the University of York's Super Advanced Research Computing Cluster (Viking). This is an extended journal version of (Dale et al. 2019a), a UCNC 2019 conference paper, including more network sizes, longer searches, more runs, and new restrictions to the creation and alteration of networks. We also include an investigation on the effects of quality when adding extra random connections to restricted topologies.

Open Access This article is licensed under a Creative Commons Attribution 4.0 International License, which permits use, sharing, adaptation, distribution and reproduction in any medium or format, as long as you give appropriate credit to the original author(s) and the source, provide a link to the Creative Commons licence, and indicate if changes were made. The images or other third party material in this article are included in the article's Creative Commons licence, unless indicated otherwise in a credit line to the material. If material is not included in the article's Creative Commons licence and your intended use is not permitted by statutory regulation or exceeds the permitted use, you will need to obtain permission directly from the copyright holder. To view a copy of this licence, visit http://creativecommons. org/licenses/by/4.0/.

\section{References}

Adamatzky A (2010) Game of life cellular automata, vol 1. Springer, Berlin

Appeltant L, Soriano MC, Van der Sande G, Danckaert J, Massar S, Dambre J, Schrauwen B, Mirasso CR, Fischer I (2011) Information processing using a single dynamical node as complex system. Nat Commun 2:468

Bala A, Ismail I, Ibrahim R, Sait SM (2018) Applications of metaheuristics in reservoir computing techniques: a review. IEEE Access 6:58012-58029

Barabási AL, Albert R (1999) Emergence of scaling in random networks. Science 286(5439):509-512

Brush SG (1967) History of the Lenz-Ising model. Rev Mod Phys 39(4):883

Crutchfield JP (1994) The calculi of emergence. Phys D 75(1-3):11-54

Dale M, Miller JF, Stepney S, Trefzer MA (2016a) Reservoir computing in materio: an evaluation of configuration through evolution. In: 2016 IEEE symposium series on computational intelligence (SSCI), pp 1-8

Dale M, Miller JF, Stepney S, Trefzer MA (2016b) Evolving carbon nanotube reservoir computers. In: International conference on unconventional computation and natural computation. Springer, pp 49-61

Dale M, Miller JF, Stepney S, Trefzer MA (2017) Reservoir computing in materio: a computational framework for in materio computing. In: 2017 international joint conference on neural networks (IJCNN), pp 2178-2185. https://doi.org/10.1109/ IJCNN.2017.7966119

Dale M, Dewhirst J, O'Keefe S, Sebald A, Stepney S, Trefzer MA (2019a) The role of structure and complexity on reservoir computing quality. In: International conference on unconventional computation and natural computation. Springer, pp 52-64

Dale M, Miller JF, Stepney S, Trefzer MA (2019b) A substrateindependent framework to characterize reservoir computers. Proc R Soc A 475(2226):20180723

Deng Z, Zhang Y (2007) Collective behavior of a small-world recurrent neural system with scale-free distribution. IEEE Trans Neural Netw 18(5):1364-1375

Gallicchio C, Micheli A, Pedrelli L (2017) Deep reservoir computing: a critical experimental analysis. Neurocomputing 268:87-99

Goudarzi A, Lakin MR, Stefanovic D (2013) DNA reservoir computing: a novel molecular computing approach. In: Rondelez Y, Woods D (eds) DNA computing and molecular programming. Springer, Berlin, pp 76-89

Harvey I (2009) The microbial genetic algorithm. In: European conference on artificial life. Springer, pp 126-133

Jaeger H (2001) Short term memory in echo state networks. Technical report GMD 152, GMD-Forschungszentrum Informationstechnik

Kawai Y, Park J, Asada M (2019) A small-world topology enhances the echo state property and signal propagation in reservoir computing. Neural Netw 112:15-23

Lavis DA (2015) Equilibrium statistical mechanics of lattice models. Springer, Berlin

Legenstein R, Maass W (2007) Edge of chaos and prediction of computational performance for neural circuit models. Neural Netw 20(3):323-334

Lehman J, Stanley KO (2008) Exploiting open-endedness to solve problems through the search for novelty. In: ALife XI, pp 329-336

Lloyd S (2000) Ultimate physical limits to computation. Nature 406(6799): 1047 
Lukoševičius M (2012) A practical guide to applying echo state networks. In: Orr GB, Müller KR (eds) Neural networks: tricks of the trade. Springer, Berlin, pp 659-686

Lukoševičius M, Jaeger H (2009) Reservoir computing approaches to recurrent neural network training. Comput Sci Rev 3(3):127-149

Ortín S, Soriano MC, Pesquera L, Brunner D, San-Martín D, Fischer I, Mirasso C, Gutiérrez J (2015) A unified framework for reservoir computing and extreme learning machines based on a single time-delayed neuron. Sci Rep 5:14945

Paquot Y, Duport F, Smerieri A, Dambre J, Schrauwen B, Haelterman M, Massar S (2012) Optoelectronic reservoir computing. Sci Rep 2:287

Pearson JE (1993) Complex patterns in a simple system. Science 261(5118):189-192

Pugh JK, Soros LB, Stanley KO (2016) Quality diversity: a new frontier for evolutionary computation. Front Robot AI 3:40

Rodan A, Tiňo P (2010) Simple deterministically constructed recurrent neural networks. In: International conference on intelligent data engineering and automated learning. Springer, pp 267-274
Rodan A, Tiňo P (2011) Minimum complexity echo state network. IEEE Trans Neural Netw 22(1):131-144

Schrauwen B, Verstraeten D, Van Campenhout J (2007) An overview of reservoir computing: theory, applications and implementations. In: Proceedings of the 15th European symposium on artificial neural networks. Citeseer

Stepney S (2008) The neglected pillar of material computation. Phys D Nonlinear Phenom 237(9):1157-1164

Tanaka G, Yamane T, Héroux JB, Nakane R, Kanazawa N, Takeda S, Numata H, Nakano D, Hirose A (2019) Recent advances in physical reservoir computing: a review. Neural Netw 115:100-123

Verstraeten D, Schrauwen B, D'Haene M, Stroobandt D (2007) An experimental unification of reservoir computing methods. Neural Netw 20(3):391-403

Watts DJ, Strogatz SH (1998) Collective dynamics of 'small-world' networks. Nature 393(6684):440

Publisher's Note Springer Nature remains neutral with regard to jurisdictional claims in published maps and institutional affiliations. 